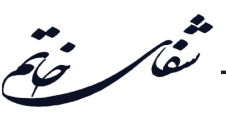

\title{
The Effect of Transcranial Direct Stimulation on Balance in Men with Schizophrenic and Depressive Disorder
}

\section{Razieh Khanmohammadi ${ }^{1}$, Mahmood Sheikh ${ }^{1 *}$, Fazlolah Bagherzadeh ${ }^{1}$, Davood Hoomanian ${ }^{1}$, Daryoosh Khajavi ${ }^{2}$}

${ }^{1}$ Department of Motor Behavior, University of Tehran, Tehran, Iran

${ }^{2}$ Department of Motor Behavior, Arak University, Arak, Iran

\section{ABSTRACT}

Introduction: Rehabilitation interventions contribute to the recovery of impaired postural control. A promising strategy to improve the efficacy of rehabilitation is transcranial direct current stimulation. We investigated whether stimulating the primary motor cortex using transcranial direct current stimulation could affect postural control in schizophrenia patients with depression. Materials and Methods: 21 men with schizophrenia and depression (mean age: $48 \pm 11 / 9$ ) were selected based on inclusion and exclusion criteria and were homogeneously assigned to the stimulation and control groups. The transcranial direct current stimulation group received $2 \mathrm{~mA}$ stimulation for $15 \mathrm{~min}$ during 12 weeks over the right and left primary motor cortices. Data of the pre-and post-test studies of standing on the dominant leg with opened and closed eyes were collected. Results: Significant differences in static balance with opened and closed eyes and dynamic balance were observed between the experimental and control groups. Conclusion: The results provide preliminary evidence indicating that transcranial direct current stimulation of the primary cortex is a safe noninvasive tool to improve s tatic balance in schizophrenia patients with depression.

\section{*Corresponding Author: Mahmood Sheikh}

Keywords:

1. Schizophrenia

2. Transcranial Direct Current Stimulation

3. Depression

E-mail: prosheikh@yahoo.com 
اثربخشى تحريك الكتريكى مستقيم فراجمجمهاي بر تعادل بيماران اسكيزوفرنى مرد با اختلال

راضيه خانمحمدى'، محمود شيخ "، فضلالله باقرزاده'، داود حومنيان'، داريوش خواجوى'

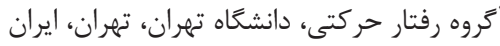

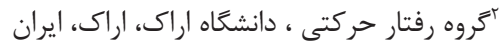

بذيرش: ا مهر و99|

اطلاعات مقاله:

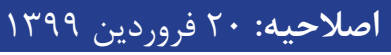

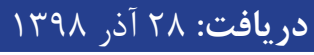

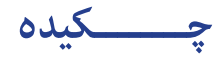

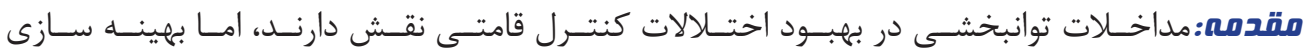

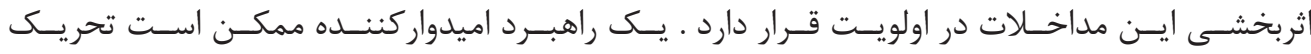

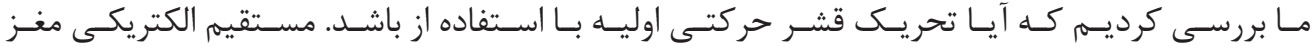

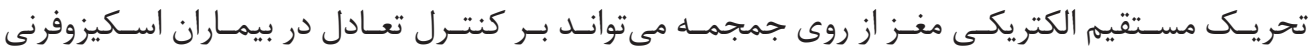

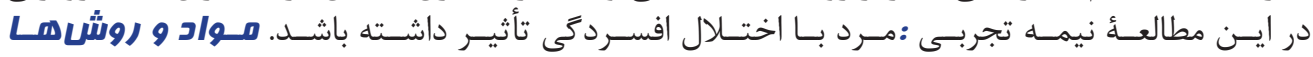

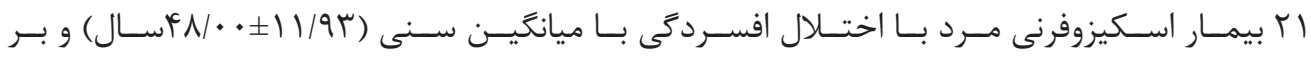

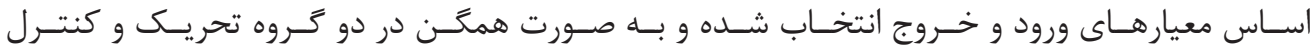

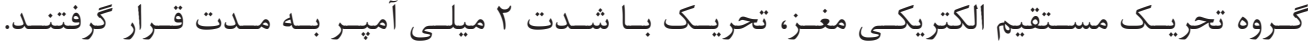

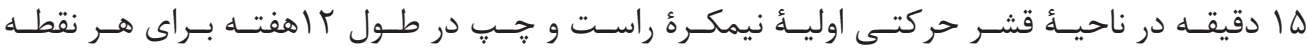

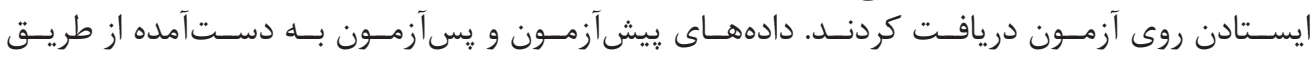

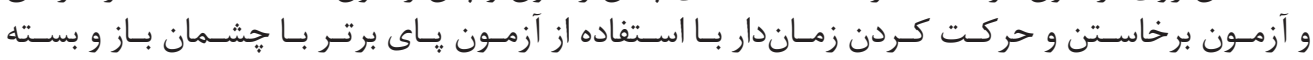

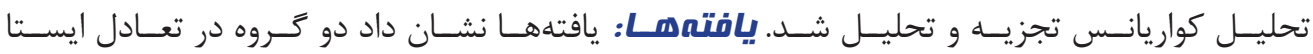

وازمهاى كليدى:

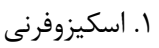

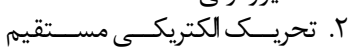

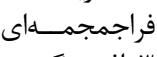

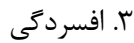

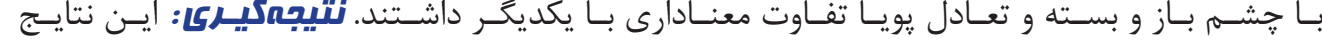

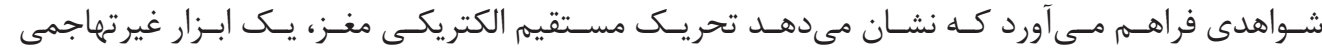

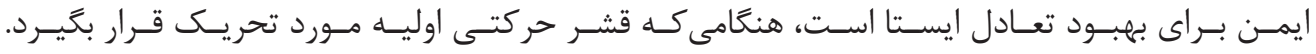

$$
\text { *ويسنده مسئول: محمود شيخ }
$$

prosheikh@yahoo.com يست الكترونيك 


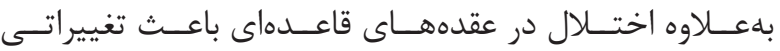

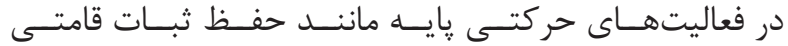

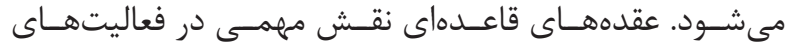

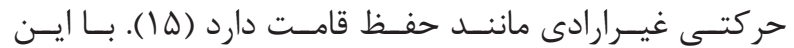

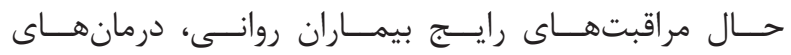

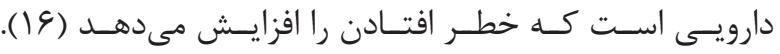

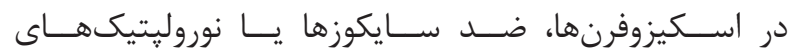

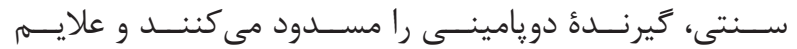

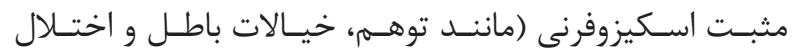

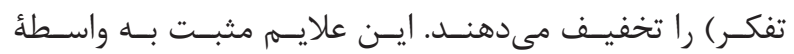

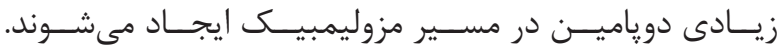

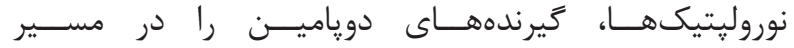

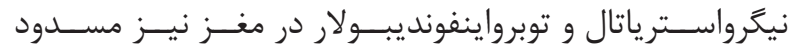

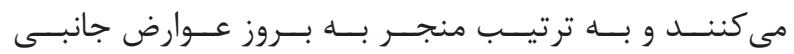

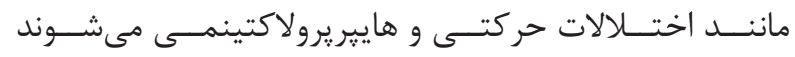

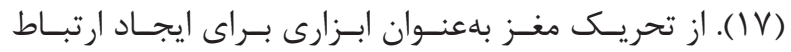

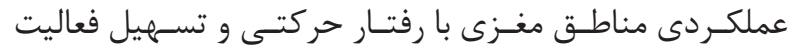

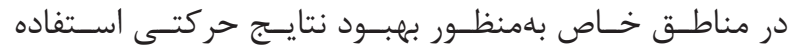

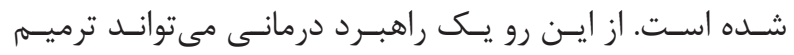

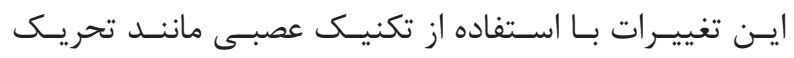

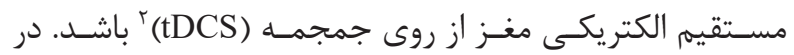

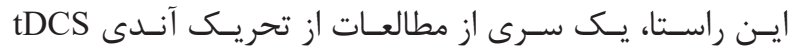

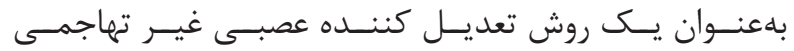

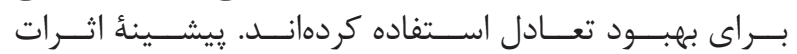
بـCS

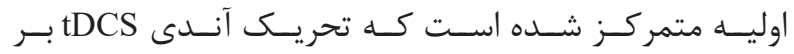

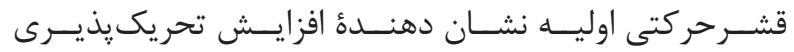

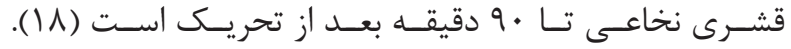

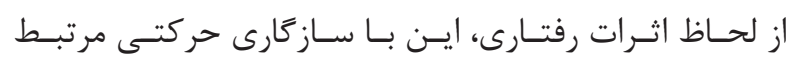

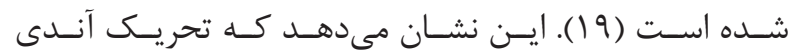

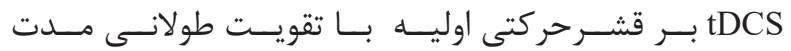

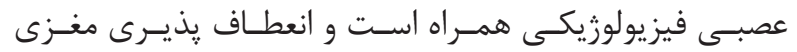

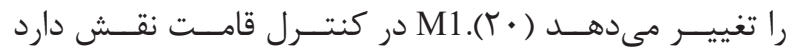

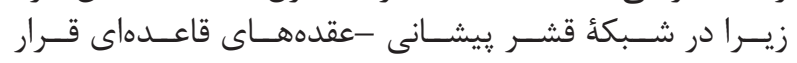

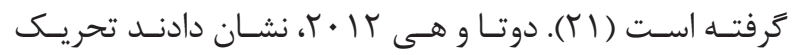

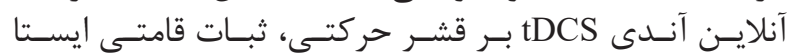

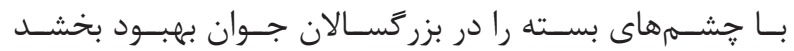

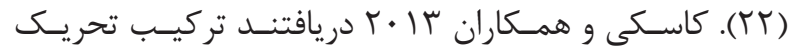

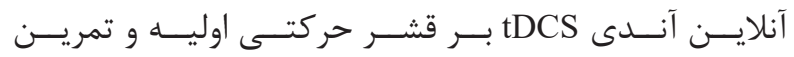

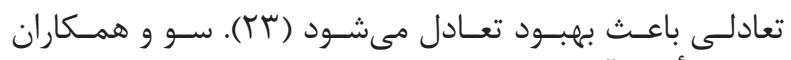

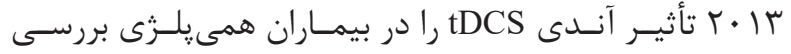

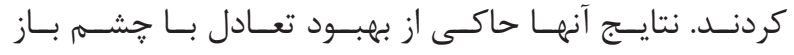

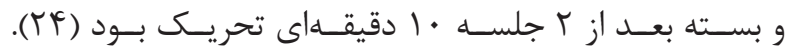

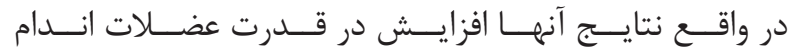

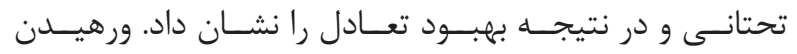

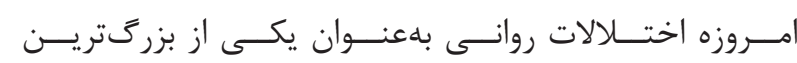

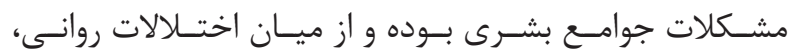

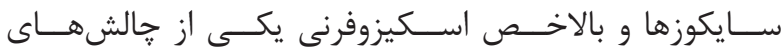

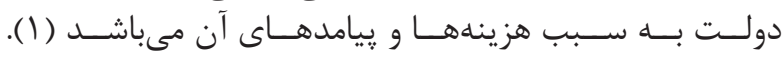

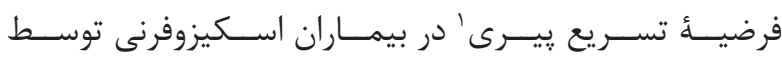

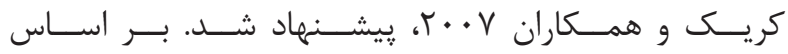

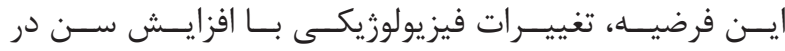

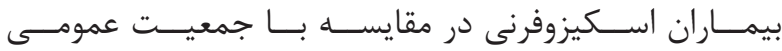

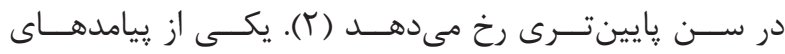

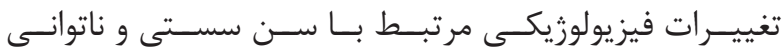

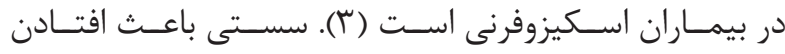

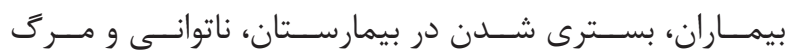

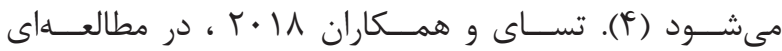

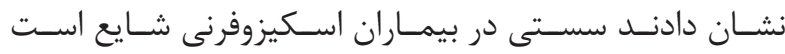

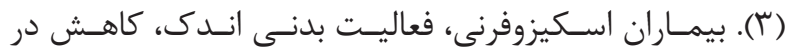

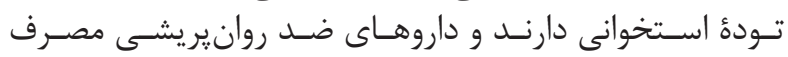

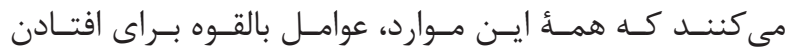

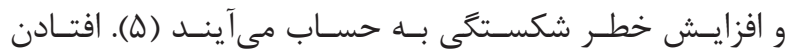

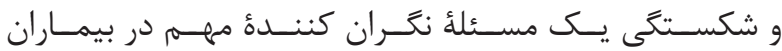

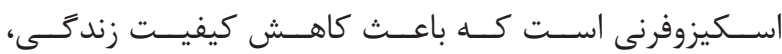

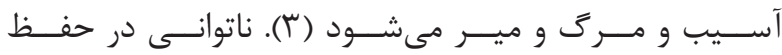

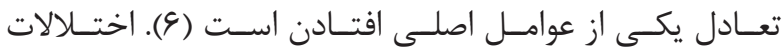

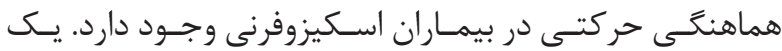

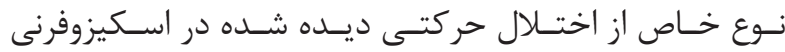

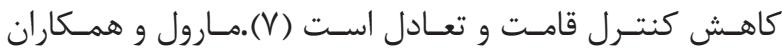

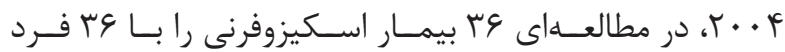

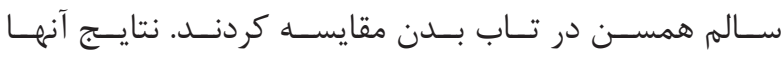

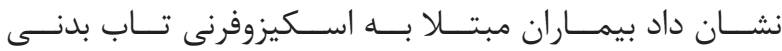

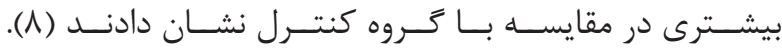

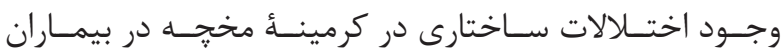

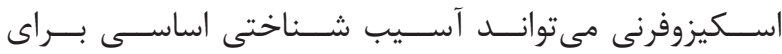

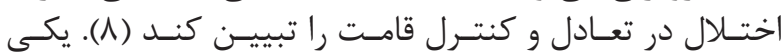

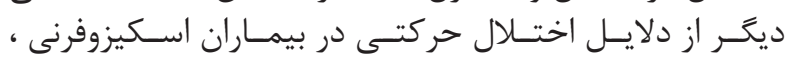

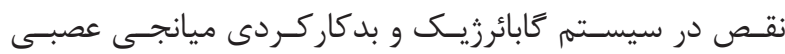

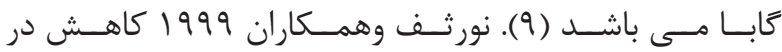

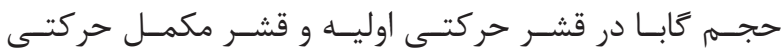

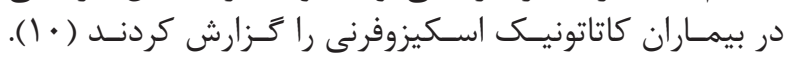
برخـى مطالعـات كاهـش فعاليـت در قشــر مكمـل حركتى

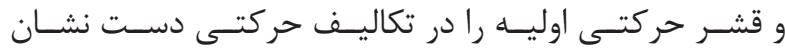

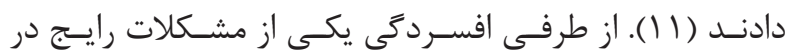

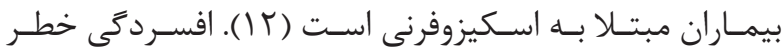

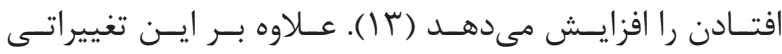

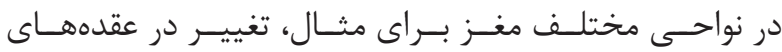

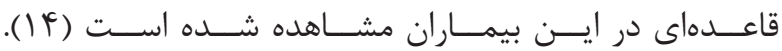




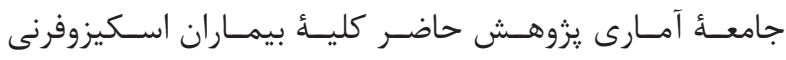

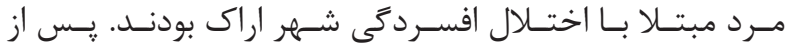

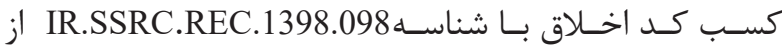

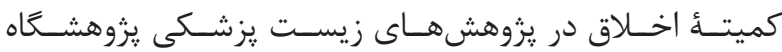

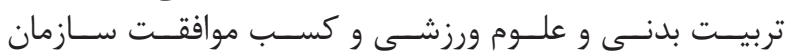

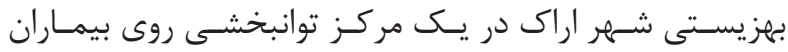

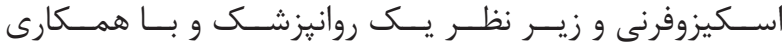

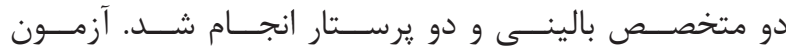

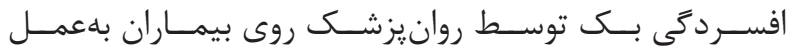

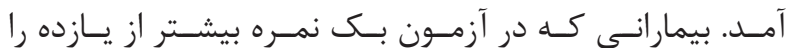

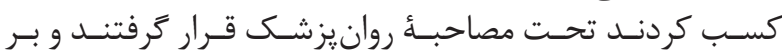

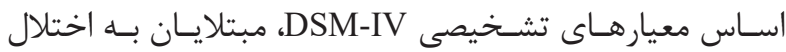

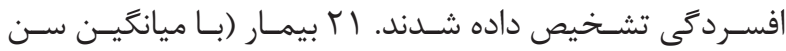

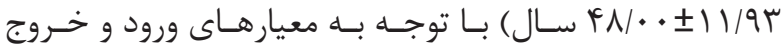

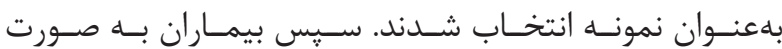

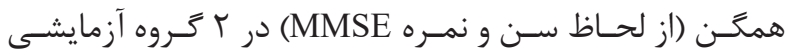

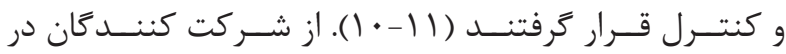

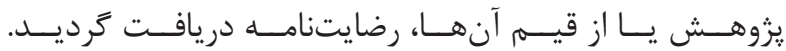

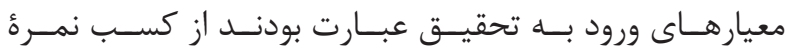

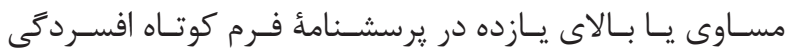

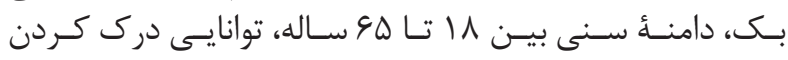

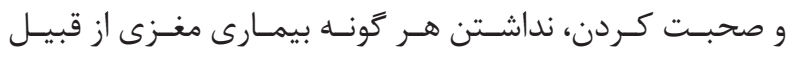

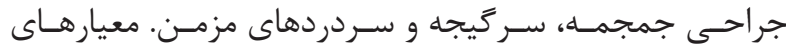

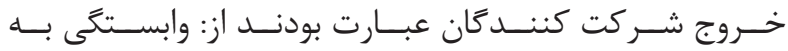

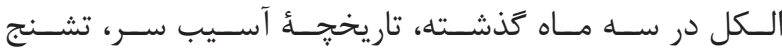

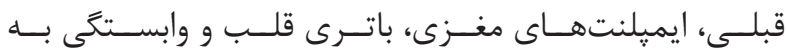

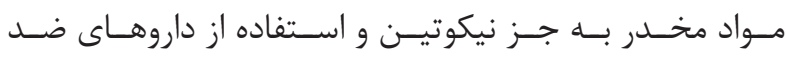

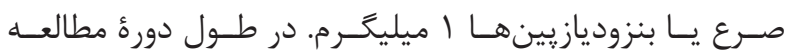

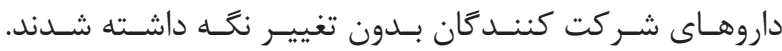

\section{يرسشنامهُ معاينة مختصر وضعيت ذهنى}

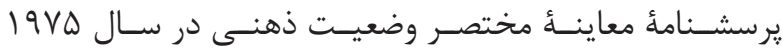

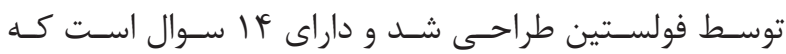

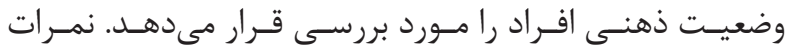

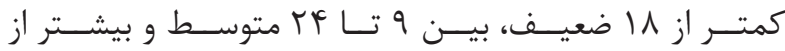

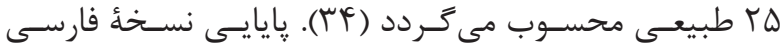

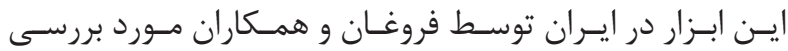

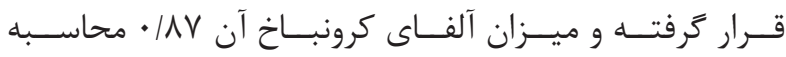

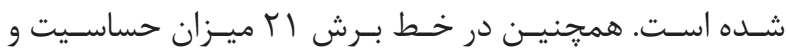

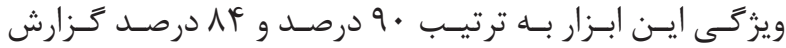

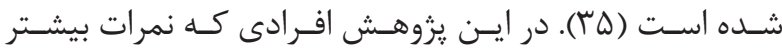

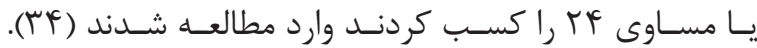

\section{ير سشنامئ فرم كوتاه افسردحى بك}

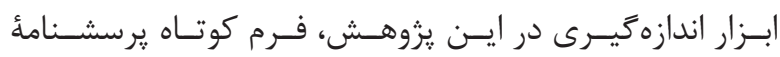

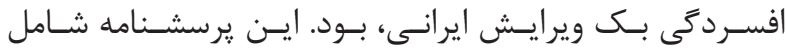

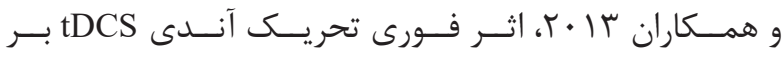

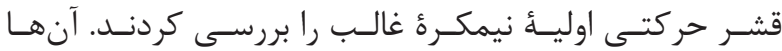

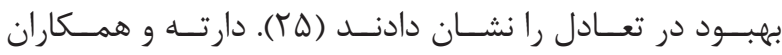

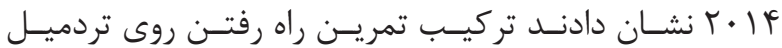

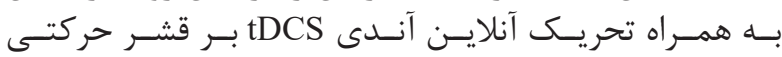

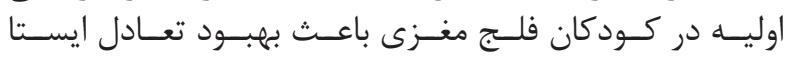

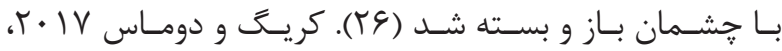

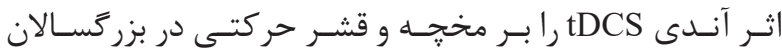

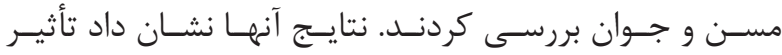

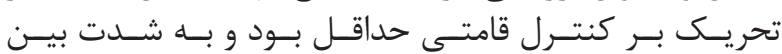

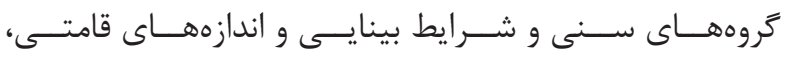

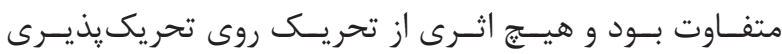

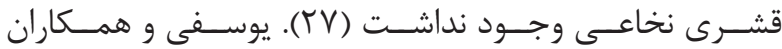

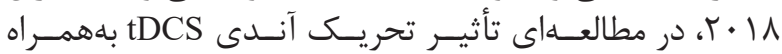

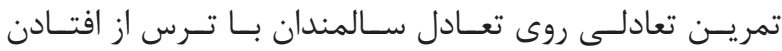

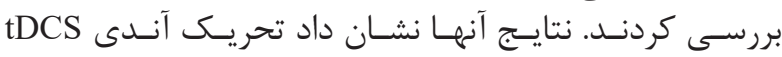

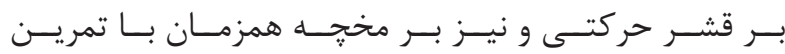

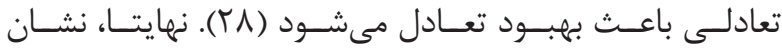

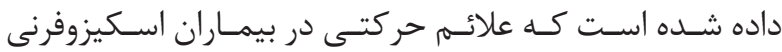

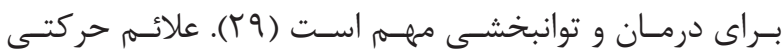

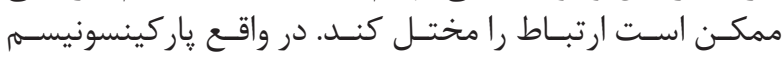

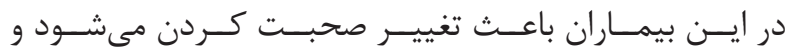

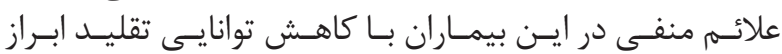

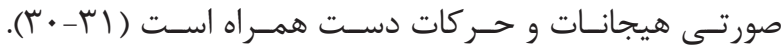

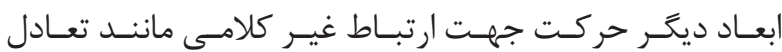

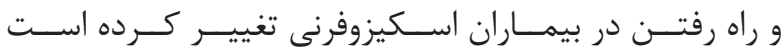

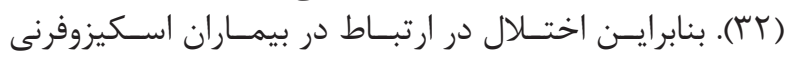

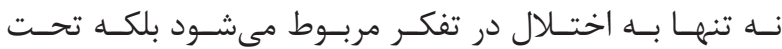

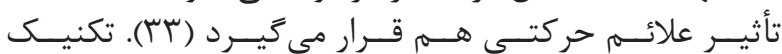

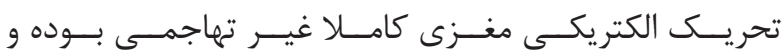

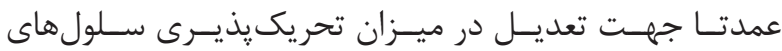

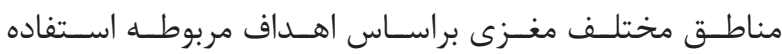

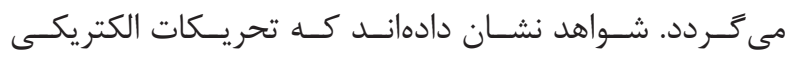

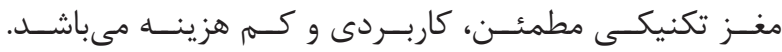

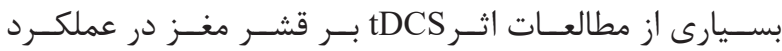

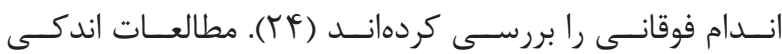

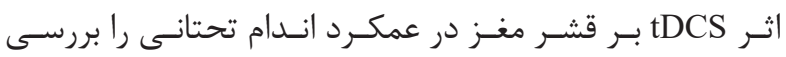

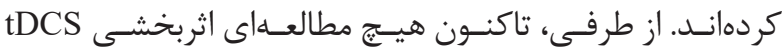

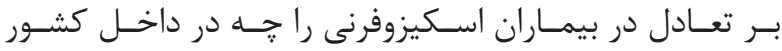

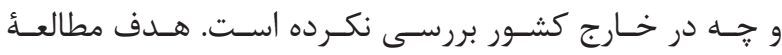

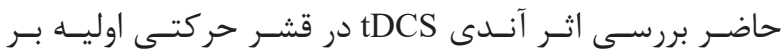

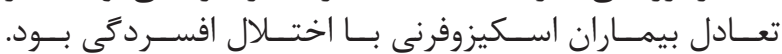

مواد و روشها

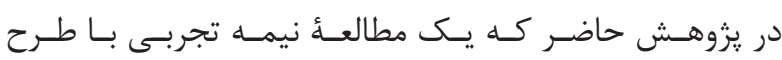

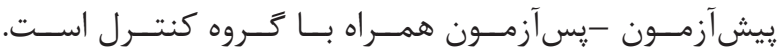




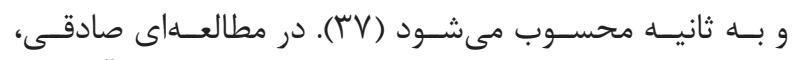

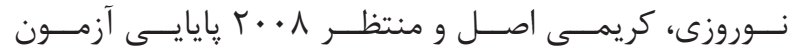

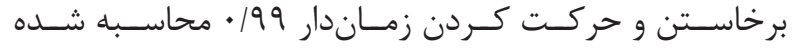

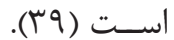

\section{تحريك مستقيم الكتريكى مغز از روى جمجمه}

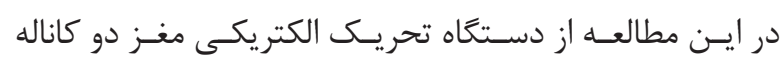

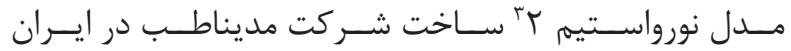

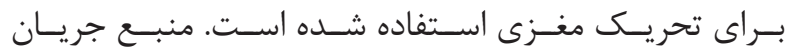

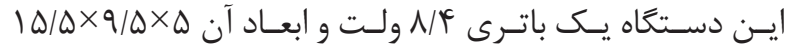

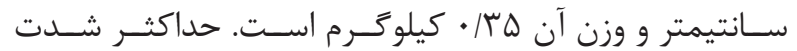

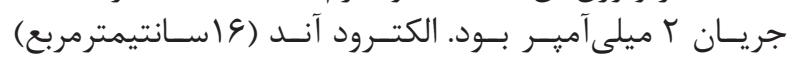

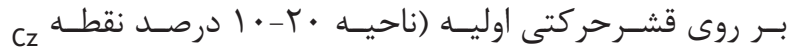

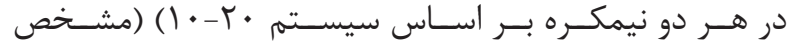

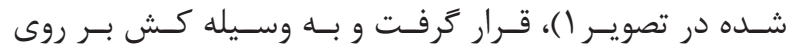

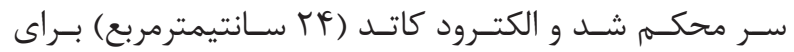

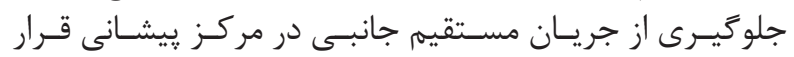

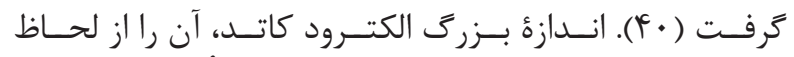

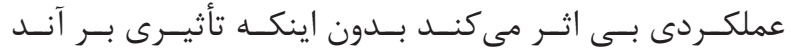

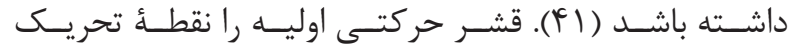

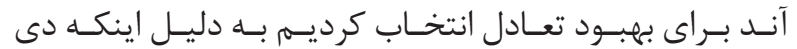

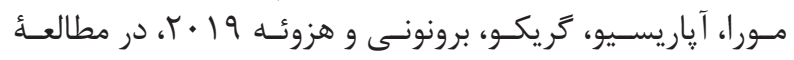

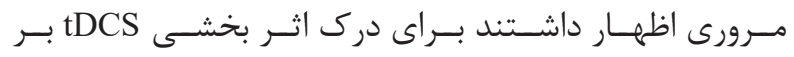

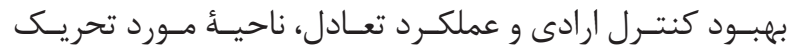

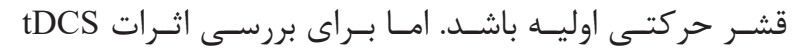

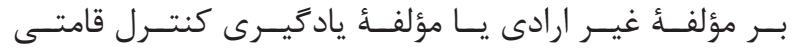

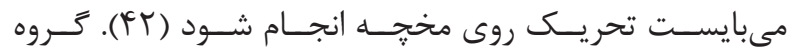

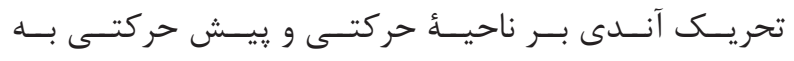

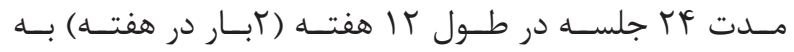

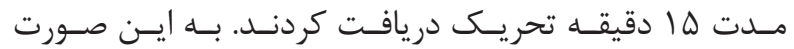

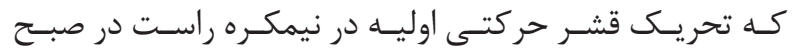

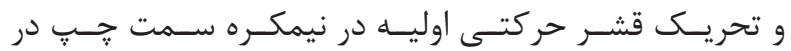

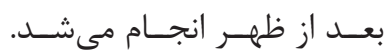

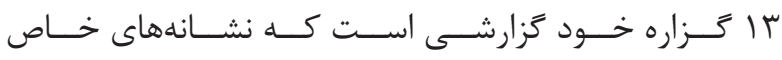

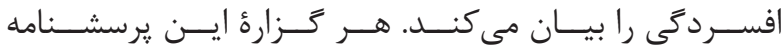

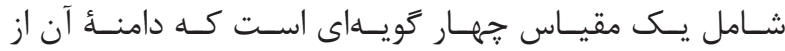

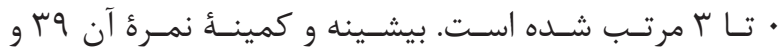

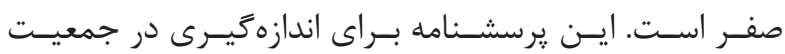

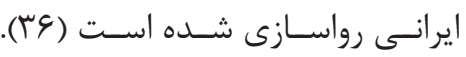
تعادل ايستا

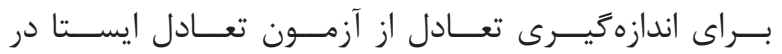

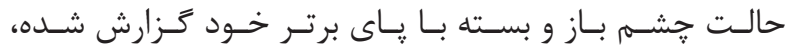

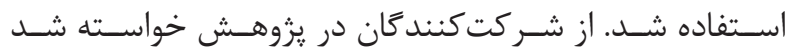

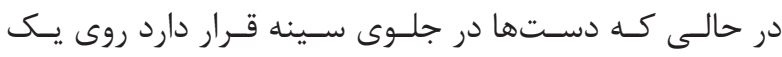

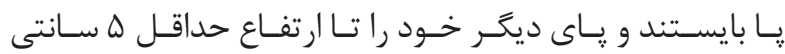

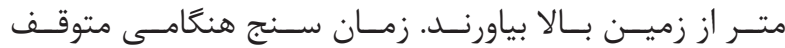

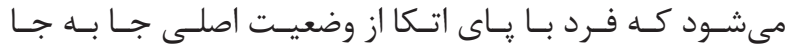

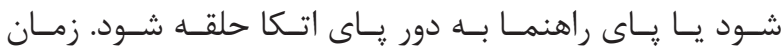

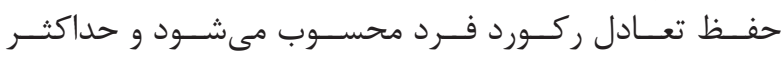

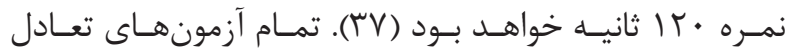

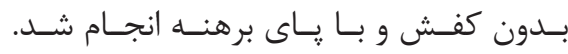

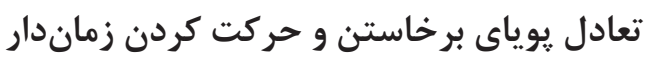

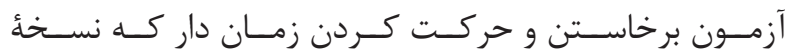

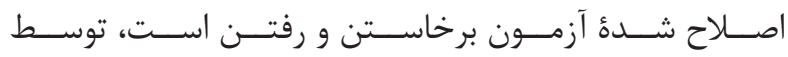

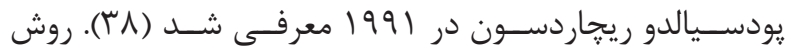

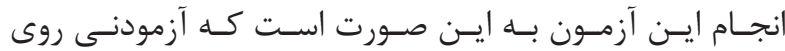

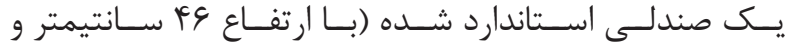

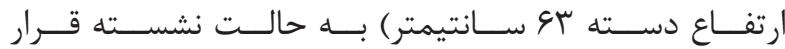

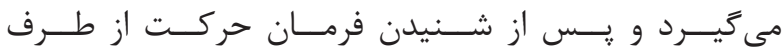

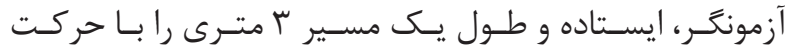

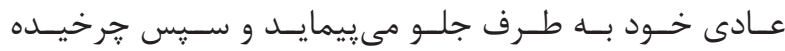

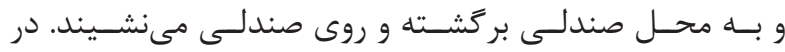

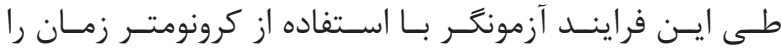

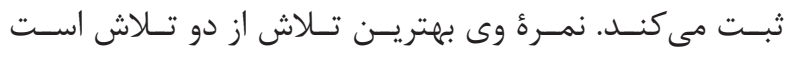

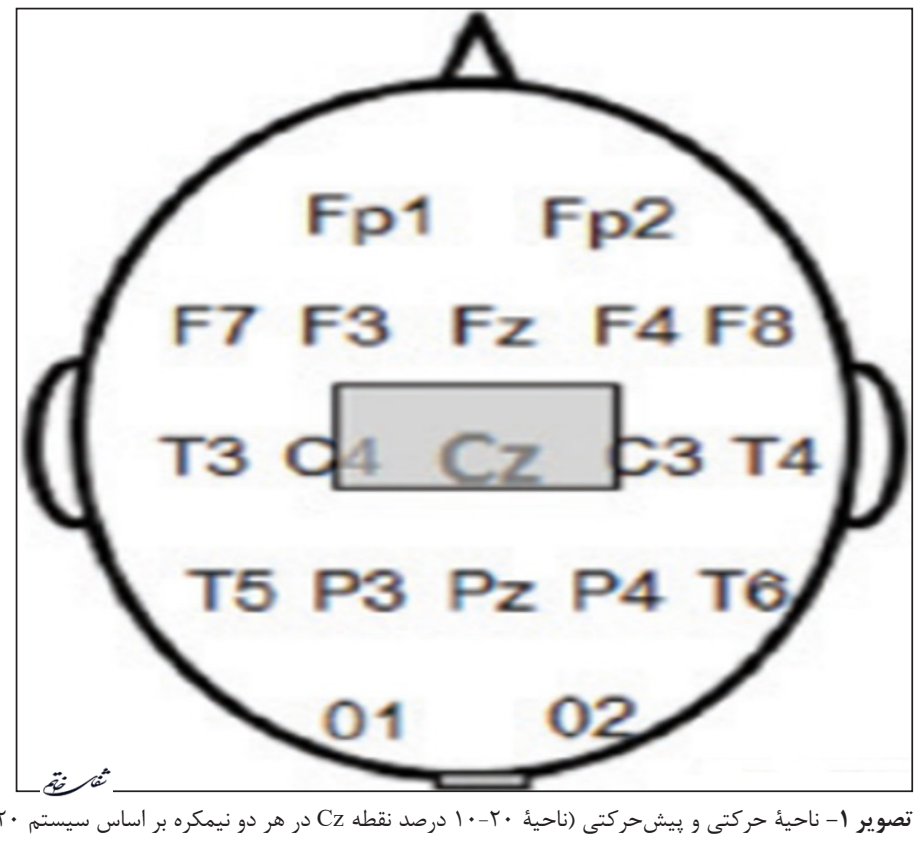

${ }^{3}$ Neurostism 2 


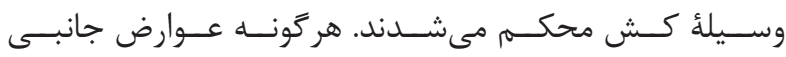

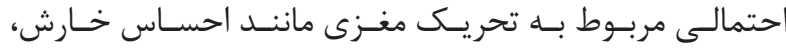

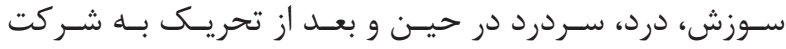

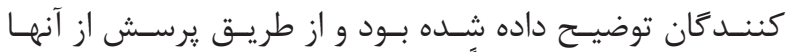

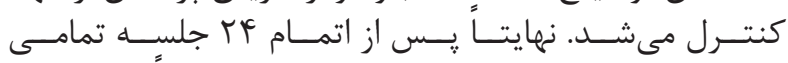

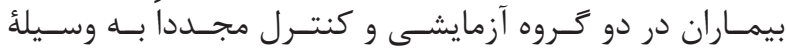

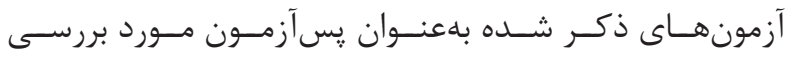

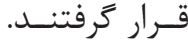
روش آمارى

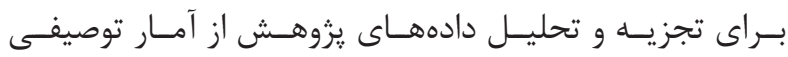

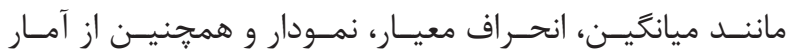

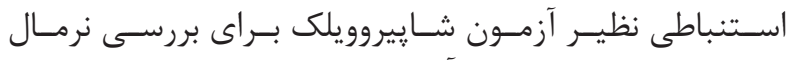

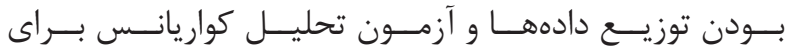

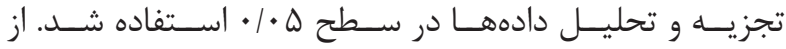

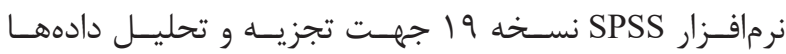

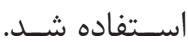

بافته ها

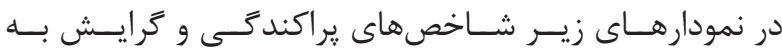

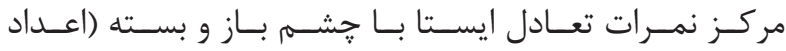

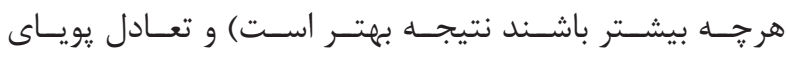

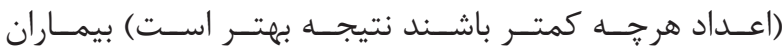

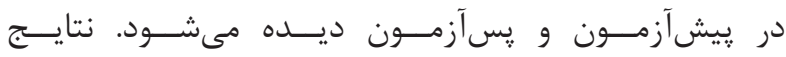

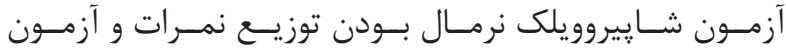

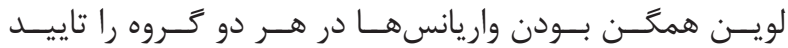

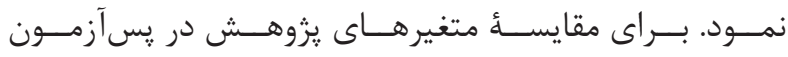

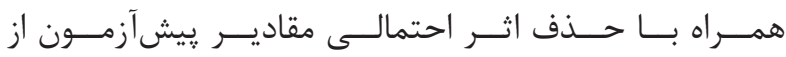

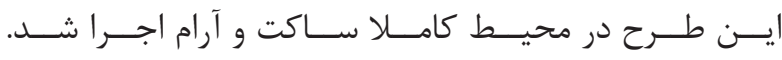

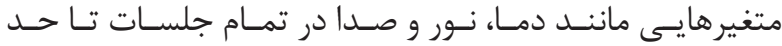

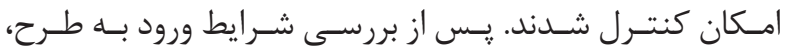

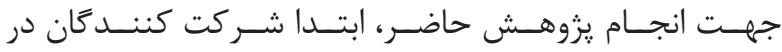

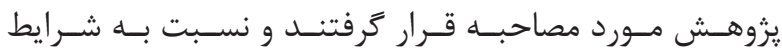

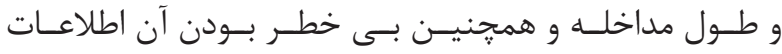

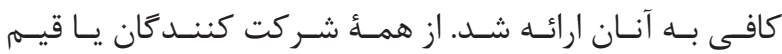

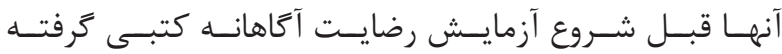

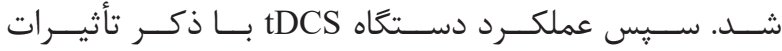

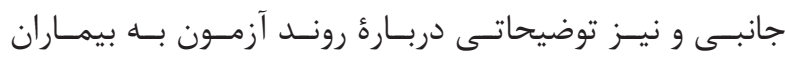

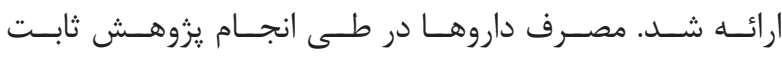

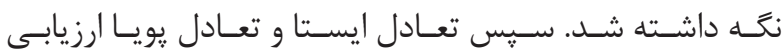

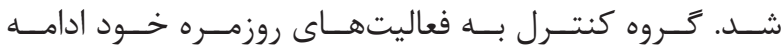

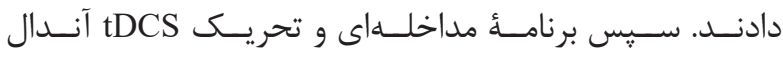

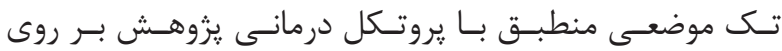

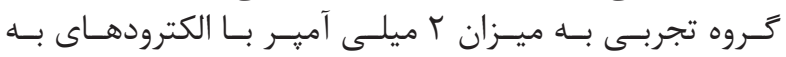

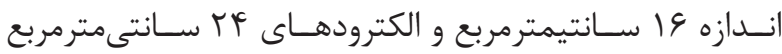

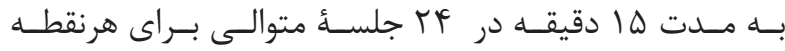

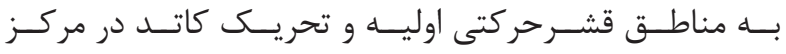

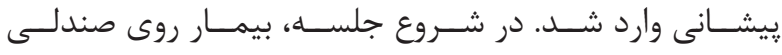

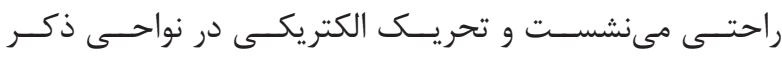

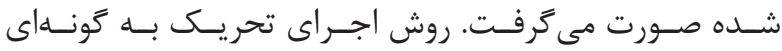

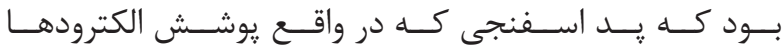

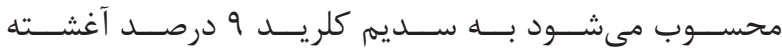

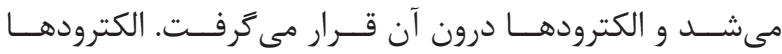

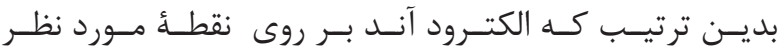

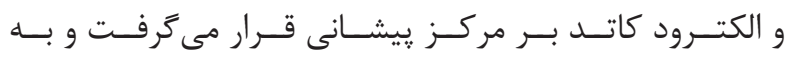

\section{تعادل با جشم باز}

\section{ثانيه}

\section{if \\ if}

ir

$1 \cdot$

$\wedge$

$q$

r

r

क

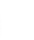

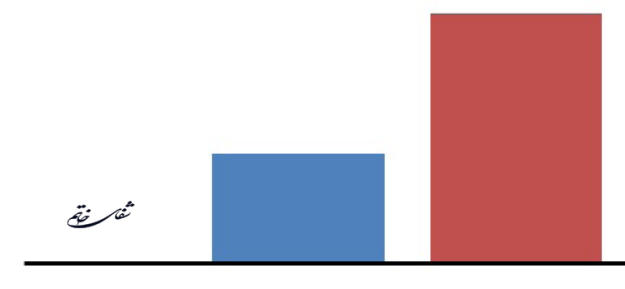

بيش آزمون

يس آزمون 


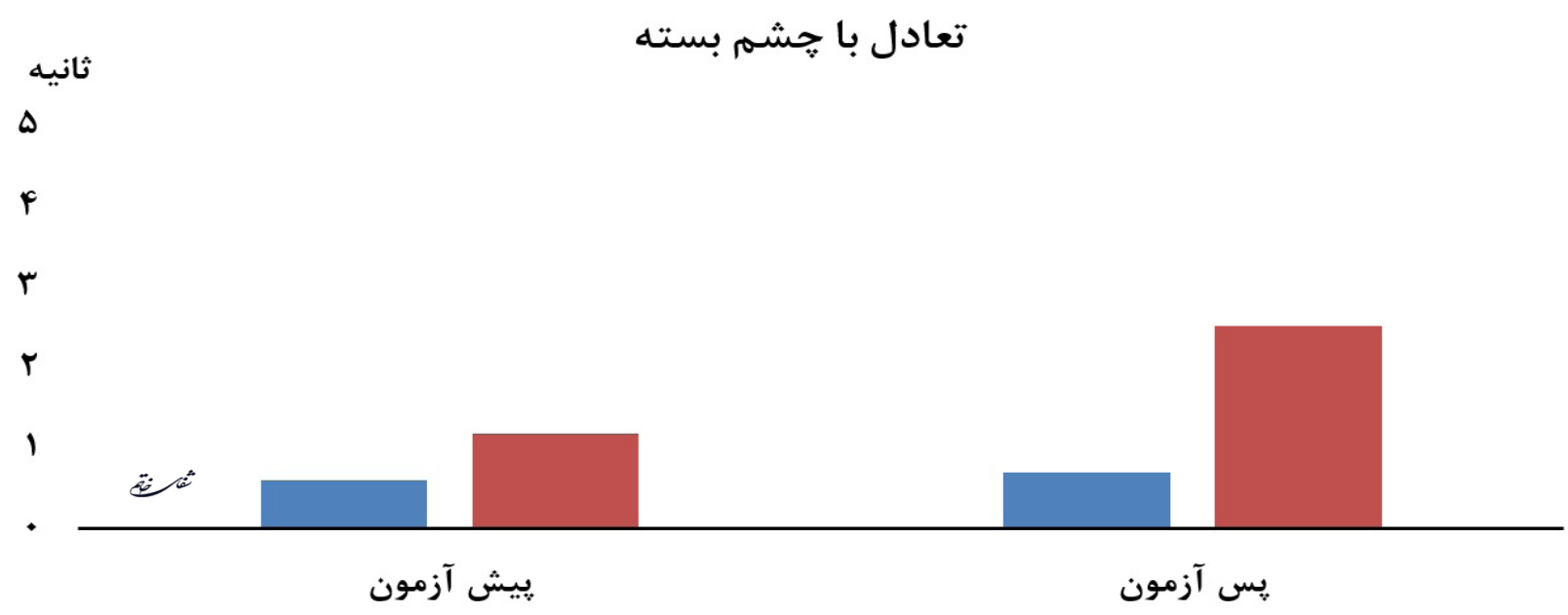

" تجربى ص كنترل

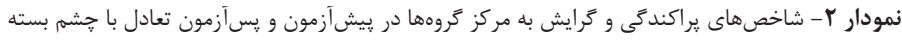

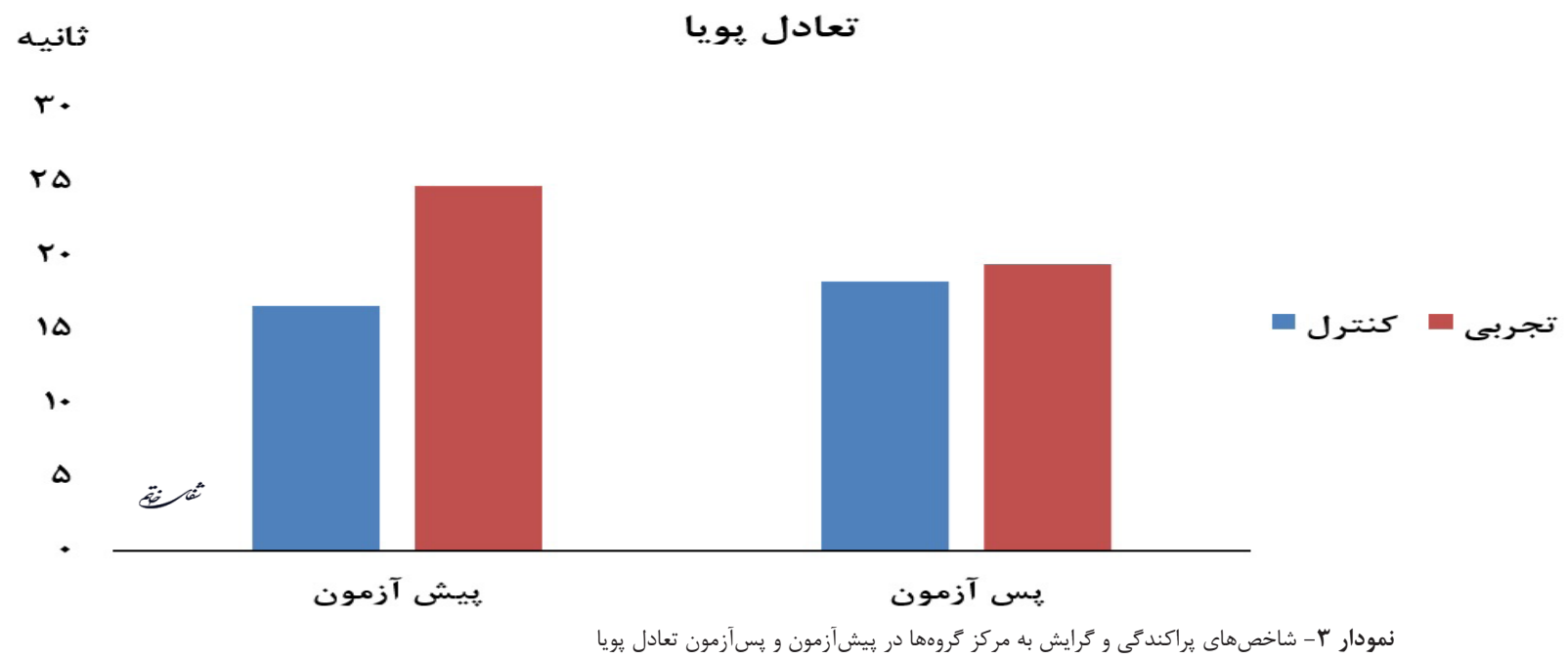

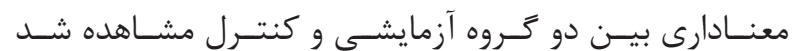

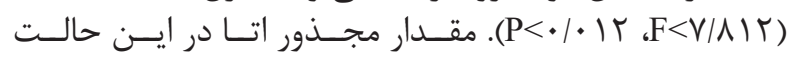

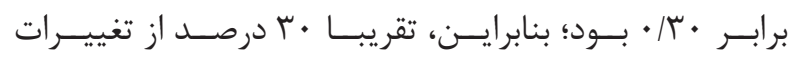

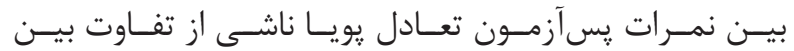

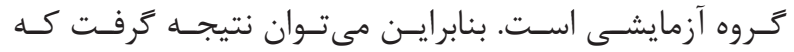

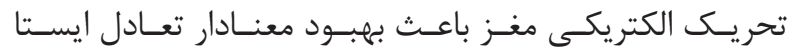

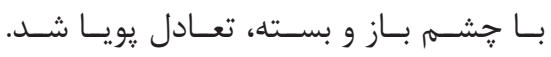

$$
\text { بحث و نتيجه }
$$

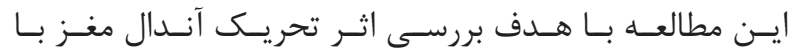

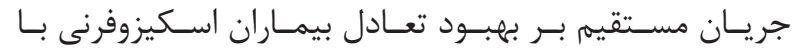

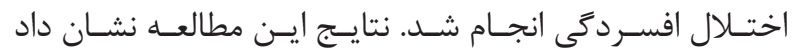

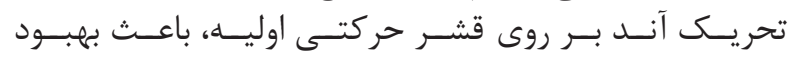

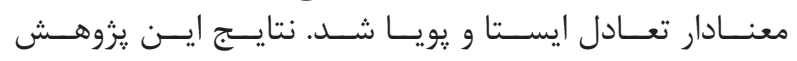

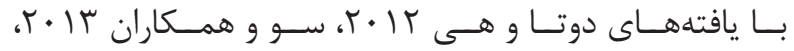

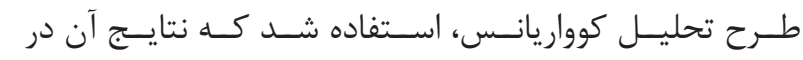

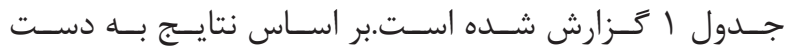

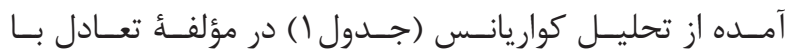

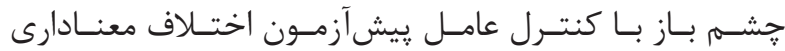

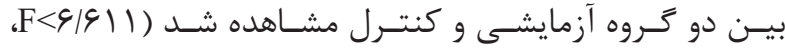

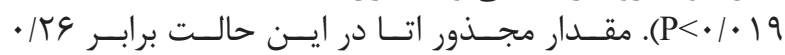

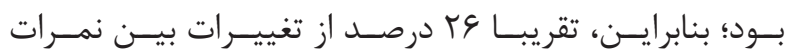

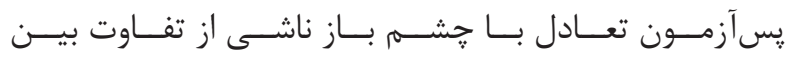

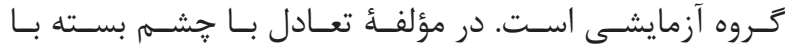

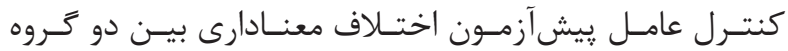

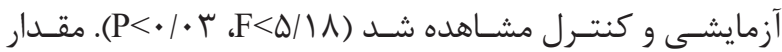

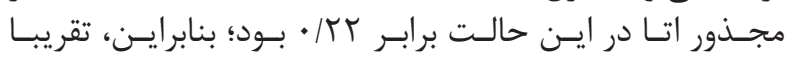

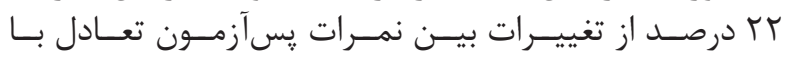

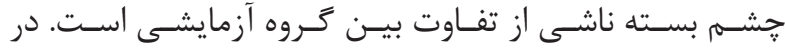

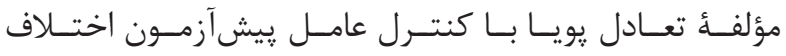




\begin{tabular}{|c|c|c|c|c|c|c|c|}
\hline الندازه اثر & سطح معنادارى & $\mathrm{F}$ & ميانَين مجنورات & درجات آزادى & مجموع مجلذورات & منبع تنييرات & متفير \\
\hline & $* 1.0 r$ & $\mid \mu /{ }^{2} y$ & TMN/TVG & 1 & TQN/TVG & يَيش أزمون & \multirow{3}{*}{ "عادل با جشم باز } \\
\hline \multirow[t]{3}{*}{. KG } & $* 1.19$ & $4|9| 1$ & IfG/fFr & 1 & IfG/Frt & كروه & \\
\hline & & & $T r / I D$. & 11 & $r 9 N \mid 994$ & خطا & \\
\hline & $* 1 . .9$ & $N \mid 9 \mathrm{VY}$ & 19/1Q" & 1 & $19 / 10 \mu$ & يشي أزمون & \multirow{3}{*}{ نعال با جشم بسته } \\
\hline \multirow[t]{3}{*}{$\cdot \pi r$} & $* / . \mu$ & $\mathrm{D} / \mathrm{l}$ & $q|g| y$ & 1 & $q|g| t$ & كروه & \\
\hline & & & $1 / 149$ & 11 & $r|\Delta|$ & خطا & \\
\hline & $* 1 . \cdot$ & $q r / \mu / f$ & NQH/GGY & 1 & NQH/GGY & يِشأزمون & \multirow{3}{*}{ "عادل يريا } \\
\hline . & $* 1.14$ & VIAIY & $V Y / Y \mu$ & 1 & $V Y / Y H$ & كروه & \\
\hline & & & $q \cdot / \mu t$ & 11 & $199 / F \Delta T$ & خطا & \\
\hline
\end{tabular}

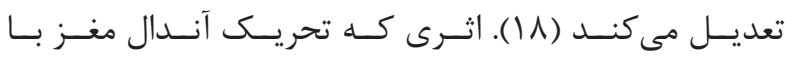

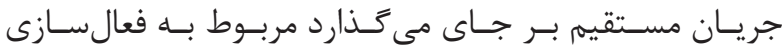

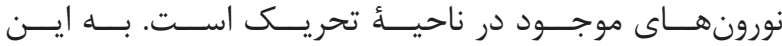

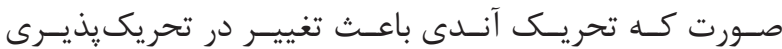

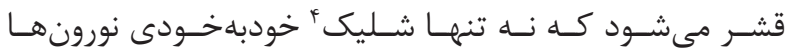

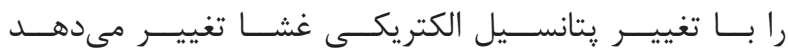

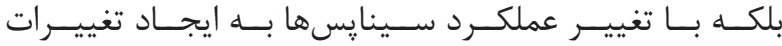

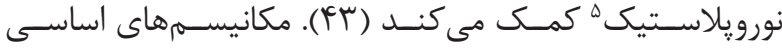

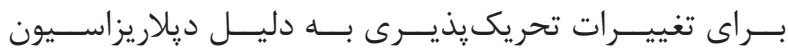

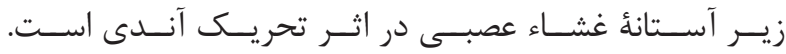

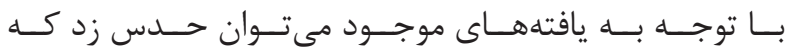

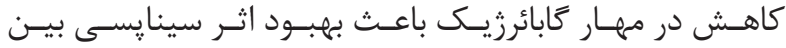

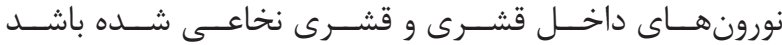

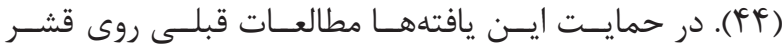

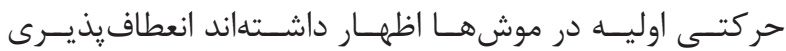

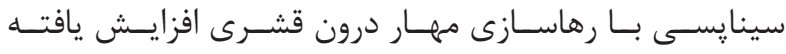

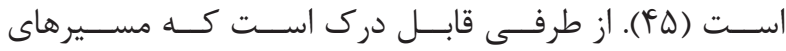

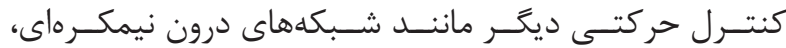

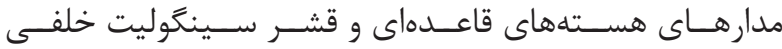

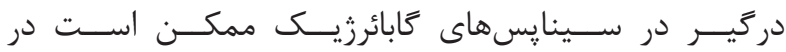

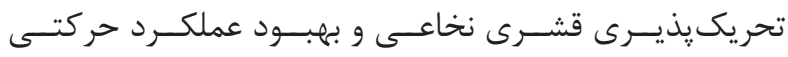

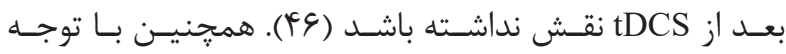

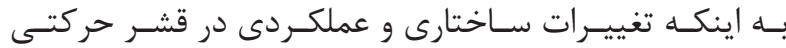

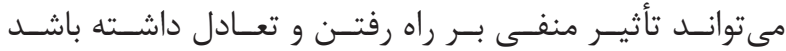

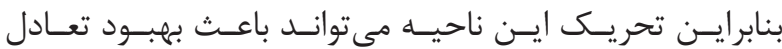

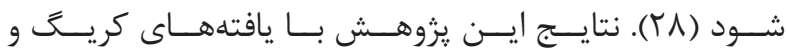

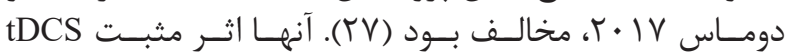

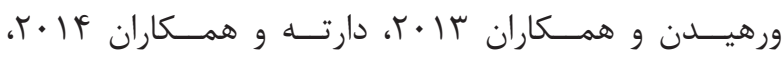

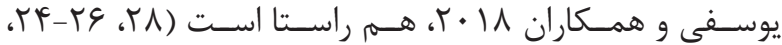

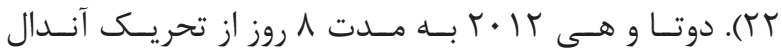

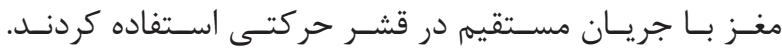

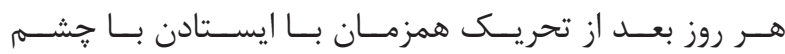

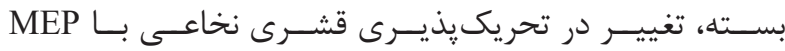

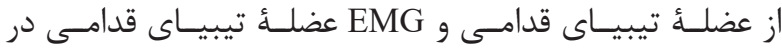

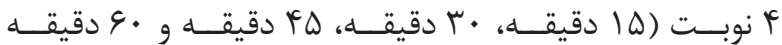

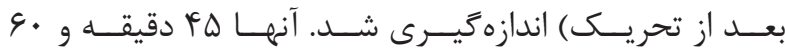

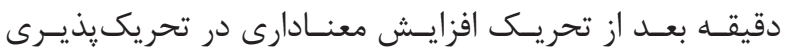

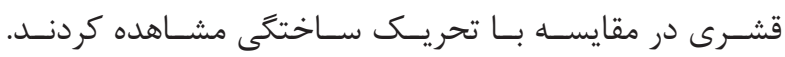

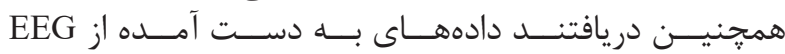

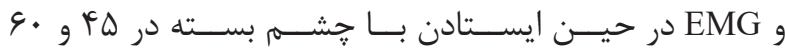

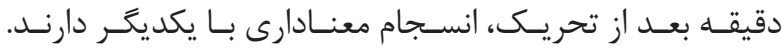

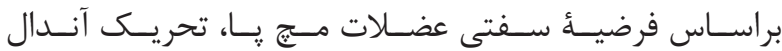

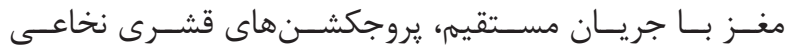

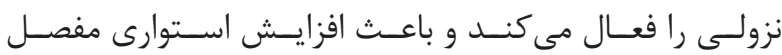

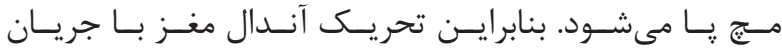

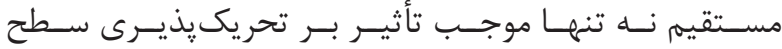

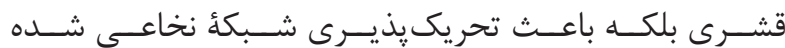

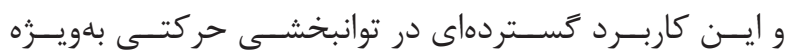

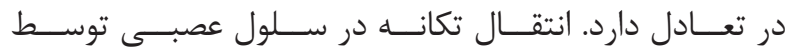

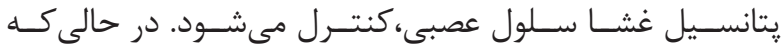

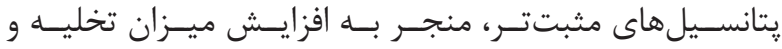

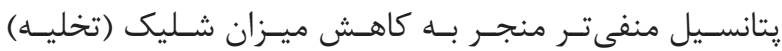

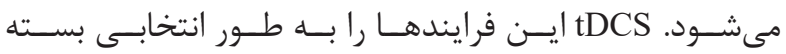

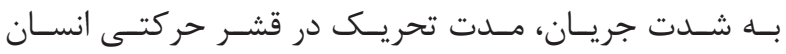




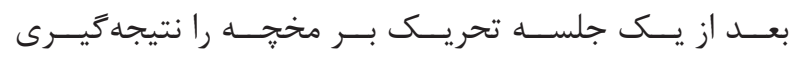

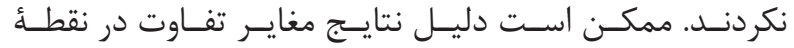

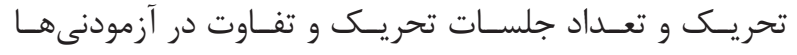

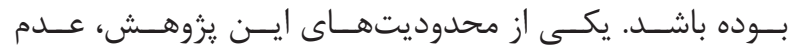

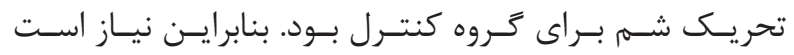

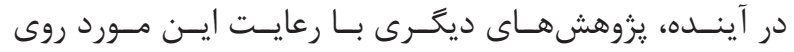

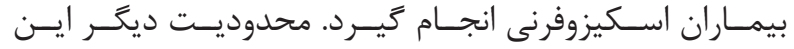

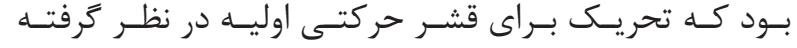

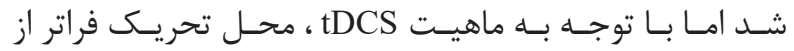

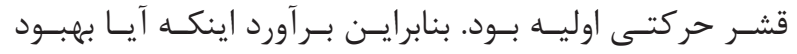

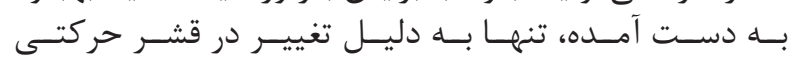

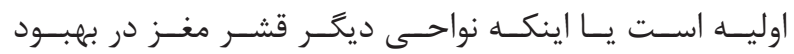

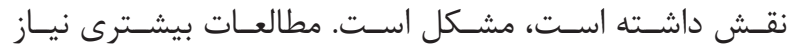

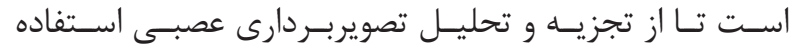

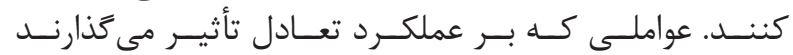

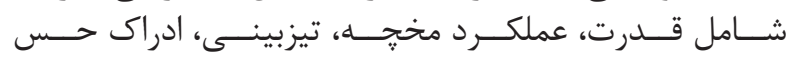

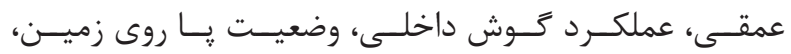

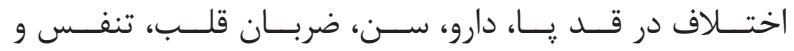

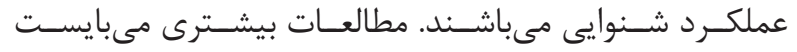

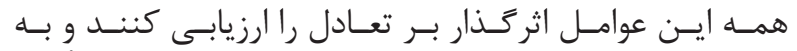

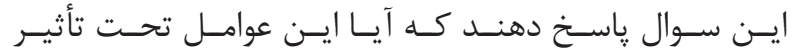
قDCS

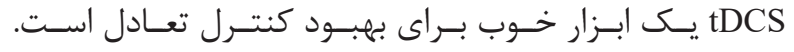

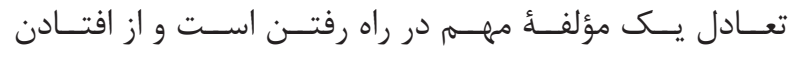

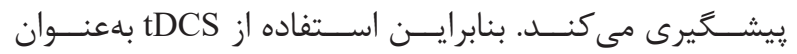

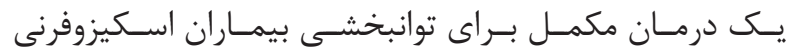

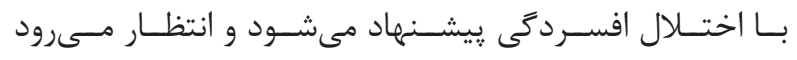

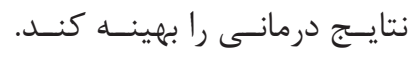

$$
\text { تشكر و قدردانى }
$$

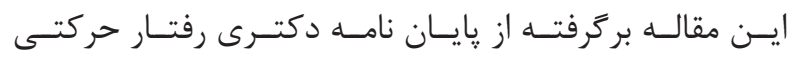

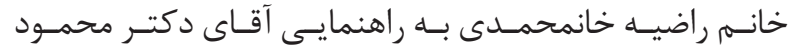

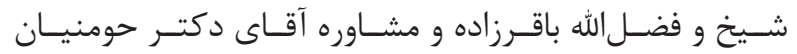

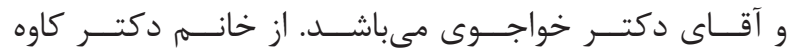

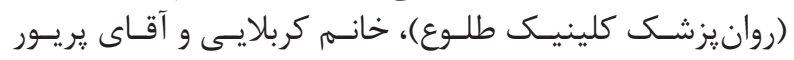

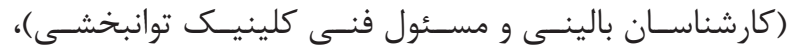

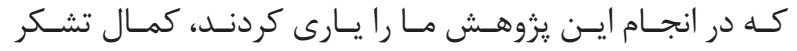
را داريسم.

1 .Kalateh-jari M, Bagheri H, Hassani M. The effect of aerobic exercise program on quality of life in schizophrenic patients. J Shahrekord Univ Med Sci. 2007; 9 (3): 28-36. [Persian]

2. Kirkpatrick B, Messias E, Harvey PD, FernandezEgea E, Bowie CR. Is schizophrenia a syndrome of accelerated aging? Schizophr Bull. 2007; 34(6): 1024-32.

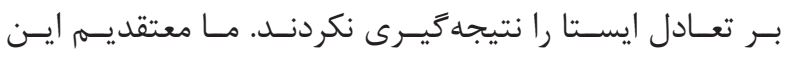

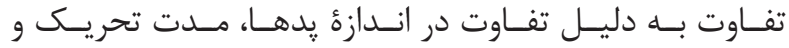

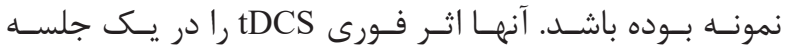

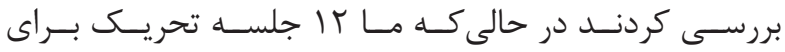

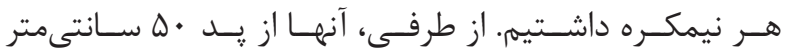

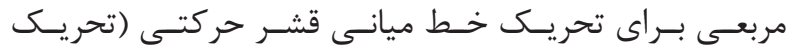

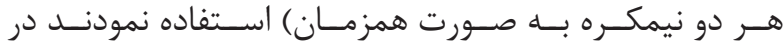

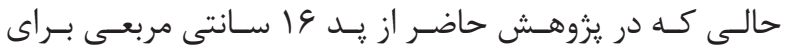

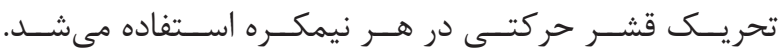

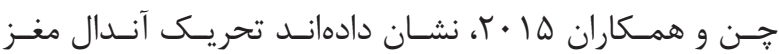

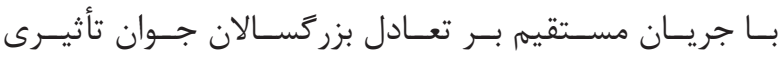

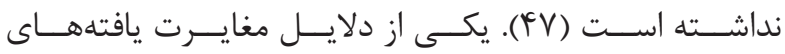

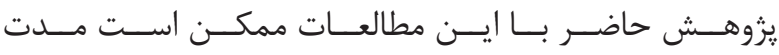

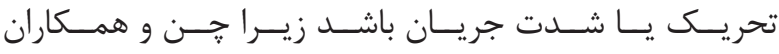

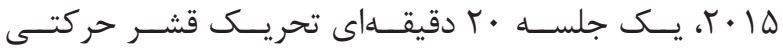

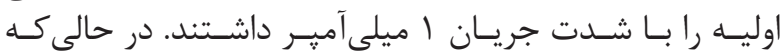

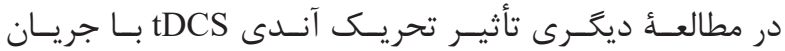

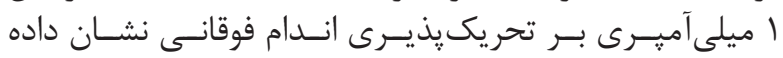

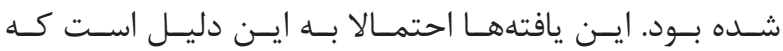

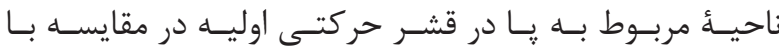

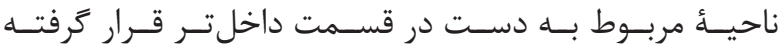

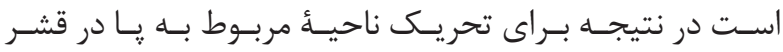

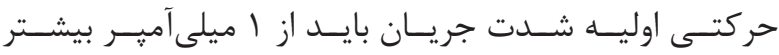

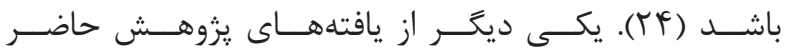

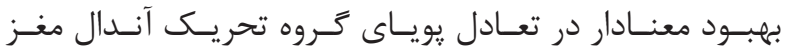

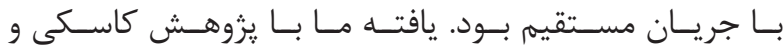

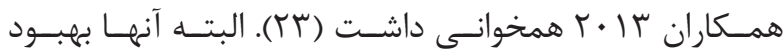

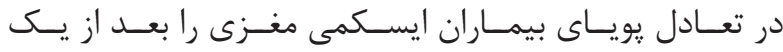

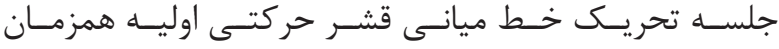

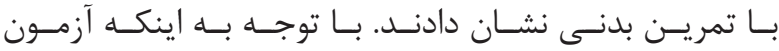

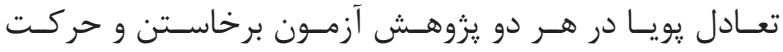

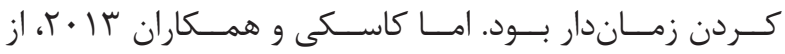

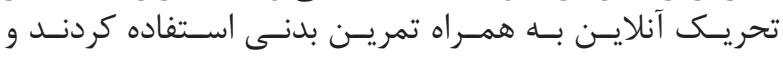

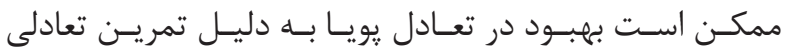

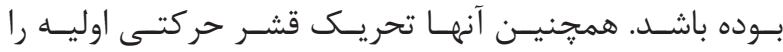

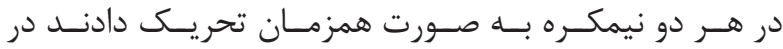

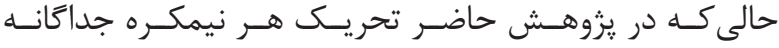

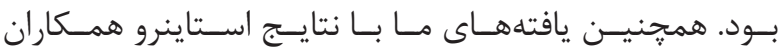

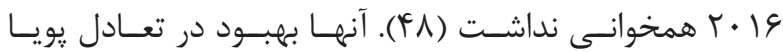

منابع

3. Tsai M-T, Lee S-M, Chen H-K, Wu B-J. Association between frailty and its individual components with the risk of falls in patients with schizophrenia spectrum disorders. Schizophr Res. 2018; 197: 138-43.

4. Bartley MM, Geda YE, Christianson TJ, Shane Pankratz V, Roberts RO, Petersen RC. Frailty and mortality outcomes in cognitively normal older people: 
sex differences in a population-based study. J Am Geriatr Soc. 2016; 64(1): 132-7.

5. Stubbs B, Gaughran F, Mitchell AJ, De Hert M, Farmer R, Soundy A, et al. Schizophrenia and the risk of fractures: a systematic review and comparative metaanalysis. Gen Hosp Psychiatry. 2015; 37(2): 126-133.

6. Mirmoezzi M, Amini M, Khaledan A, Khorshidi D. Effect of 8-week of selected aerobic exercise on static and dynamic balance in healthy elderly inactive men. Iranian Journal of Ageing. 2016; 11(1): 202-9 [Persian]

7. Bhattacharya A, Morgan R, Shukla R, Ramakrishanan H, Wang L. Non-invasive estimation of afferent inputs for postural stability under low levels of alcohol. Ann Biomed Eng. 1987; 15(6): 533-50.

8. Marvel CL, Schwartz BL, Rosse RB. A quantitative measure of postural sway deficits in schizophrenia Schizophr Res. 2004; 68(2-3): 363-72.

9. Northoff G. Catatonia and neuroleptic malignant syndrome: psychopathology and pathophysiology. J Neural Transm. 2002; 109(12): 1453-67.

10. Northoff G, Steinke R, Czcervenka C, Krause R, Ulrich S, Danos P, et al. Decreased density of GABA-A receptors in the left sensorimotor cortex in akinetic catatonia: investigation of in vivo benzodiazepine receptor binding. J Neurol Neurosurg Psychiatry Res. 1999; 67(4): 445-50.

11. Schröder J, Wenz F, Schad L, Baudendistel K, Knopp M. Sensorimotor cortex and supplementary motor area changes in schizophrenia: a study with functional magnetic resonance imaging. Br J Psychiatry. 1995; 167(2): 197-201.

12. Hasan A, Nitsche MA, Herrmann M, SchneiderAxmann T, Marshall L, Gruber O, et al. Impaired longterm depression in schizophrenia: a cathodal tDCS pilot study. Brain Stimul. 2012; 5(4): 475-83.

13. Prizer LP, Smith ML, Housman J, Ory MG. Depressive symptomology management and falls among middle aged and older adults. Aging \& mental health. 2016; 20(1): 13-21.

14. Drevets WC, Furey ML. Replication of scopolamine's antidepressant efficacy in major depressive disorder: a randomized, placebo-controlled clinical trial. .Biol
Psychiatry. 2010; 67(5): 432-8.

15. Visser JE, Bloem BR. Role of the basal ganglia in balance control. Neural plasticity. 2005; 12(2-3): 161-74.

16. Sterke CS, Ziere G, van Beeck EF, Looman CW, van der Cammen TJ. Dose-response relationship between selective serotonin re-uptake inhibitors and injurious falls: a study in nursing home residents with dementia. .Br J Clin Pharmacol. 2012; 73(5): 812-20.

17. Lohr J, Wisniewski A, Jeste D. Neurological aspects of tardive dyskinesia. Handbook of schizophrenia. 1986; 1: $97-119$

18. Nitsche MA, Paulus W. Excitability changes induced in the human motor cortex by weak transcranial direct current stimulation. J Physiol. 2000; 527(3): 633-9.

19. Kaski D, Quadir S, Patel M, Yousif N, Bronstein AM. Enhanced locomotor adaptation aftereffect in the "broken escalator" phenomenon using anodal tDCS. J Neurophysiol. 2012; 107(9): 2493-505.

20. Goodwill AM, Reynolds J, Daly RM, Kidgell DJ. Formation of cortical plasticity in older adults following tDCS and motor training. Front Aging Neurosci. 2013; 587.

21. Demain A, Westby GM, Fernandez-Vidal S, Karachi C, Bonneville F, Do MC, et al. High-level gait and balance disorders in the elderly: a midbrain disease? J Neurol. 2014; 261(1): 196-206.

22. Dutta A, Chugh S, editors. Effect of transcranial direct current stimulation on cortico-muscular coherence and standing postural steadiness. The 2nd IASTED International Conference on Assistive Technologies; Conf. Proc. IEEE Eng. Med. Biol. Soc; 2011: 7643-646.

23. Kaski D, Dominguez RO, Allum JH, Bronstein AM. Improving gait and balance in patients with leukoaraiosis using transcranial direct current stimulation and physical training: an exploratory study. Neurorehabil Neural Repair. 2013; 27(9): 864-71.

24. Sohn MK, Jee SJ, Kim YW. Effect of transcranial direct current stimulation on postural stability and lower extremity strength in hemiplegic stroke patients. Ann Rehabil Med. 2013; 37(6): 759.

25. Verheyden G, Purdey J, Burnett M, Cole J, Ashburn 


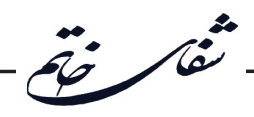

A. Immediate effect of transcranial direct current stimulation on postural stability and functional mobility in Parkinson's disease. .Mov Disord. 2013; 28(14): 2040-1.

26. Duarte NdAC, Grecco LAC, Galli M, Fregni F, Oliveira CS. Effect of transcranial direct-current stimulation combined with treadmill training on balance and functional performance in children with cerebral palsy: a double-blind randomized controlled trial. PloS one. 2014; 9(8): e105777.

27. Craig CE, Doumas M. Anodal transcranial direct current stimulation shows minimal, measure-specific effects on dynamic postural control in young and older adults: a double blind, sham-controlled study. PloS one. 2017; 12(1): e0170331.

28. Yosephi MH, Ehsani F, Zoghi M, Jaberzadeh S. Multi-session anodal tDCS enhances the effects of postural training on balance and postural stability in older adults with high fall risk: primary motor cortex versus cerebellar stimulation. Brain Stimul. 2018; 11(6): 1239-50.

29. Aubin G, Stip E, Gélinas I, Rainville C, Chapparo C. Daily activities, cognition and community functioning in persons with schizophrenia. Schizophr Res. 2009; 107(2-3): 313-8.

30. Cannizzaro MS, Cohen H, Rappard F, Snyder PJ. Bradyphrenia and bradykinesia both contribute to altered speech in schizophrenia: a quantitative acoustic study. Cogn Behav Neurol. 2005; 18(4): 206-10.

31. Park S, Matthews N, Gibson C. Imitation, simulation, and schizophrenia. Schizophr Bull. 2008; 34(4): 698-707.

32. Peralta V, Campos MS, De Jalón EG, Cuesta MJ. Motor behavior abnormalities in drug-naïve patients with schizophrenia spectrum disorders. Mov Disord. 2010; 25(8): 1068-76.

33. Kring AM, Moran EK. Emotional response deficits in schizophrenia: insights from affective science. Schizophr Bull. 2008; 34(5): 819-34.

34. Folstein MF, Folstein SE, McHugh PR. "Minimental state": a practical method for grading the cognitive state of patients for the clinician. J Psychiatr Res. 1975; 12(3): 189-98.
35. Foroughan M, JAFARI Z, SHIRIN BP, GHAEM MFZ, RAHGOZAR M. Validation of mini-mental state examination (MMSE) in the elderly population of Tehran. 2008 Adv Cogn Sci. 2008; 10(2): 29-37 [Persian].

36. Dadfar M, Kalibatseva Z. Psychometric properties of the Persian version of the short Beck Depression Inventory with Iranian psychiatric outpatients. Scientifica. 2016.

37. SUZUKI T. Thresholds of physical activities necessary for living a self-supporting life in elderly women. Osaka City Med J. 2007; 53: 53-61.

38. Podsiadlo D, Richardson S. The timed "Up \& Go": a test of basic functional mobility for frail elderly persons. J Am Geriatr Soc. 1991; 39(2): 142-8.

39. Sadeghi H, Norouzi H, Karimi Asl A, Montazer M. ]Functional Training Program Effect on Static and Dynamic Balance in Male Able-bodied Elderly[. Salmand: Iranian Journal of Ageing. 2008; 3(2): 565-571 [Persian]

40. Poortvliet P, Hsieh B, Cresswell A, Au J, Meinzer M. Cerebellar transcranial direct current stimulation improves adaptive postural control. Clin Neurophysiol. 2018; 129(1): 33-41.

41. Stagg CJ, Nitsche MA. Physiological basis of transcranial direct current stimulation. Neuroscientist. 2011; 17(1): 37-53.

42. de Moura MCDS, Hazime FA, Marotti Aparicio LV, Grecco LA, Brunoni AR, Hasue RH. Effects of transcranial direct current stimulation (tDCS) on balance improvement: a systematic review and meta-analysis. Somatosens Mot Res. 2019: 1-14.

43. Madhavan S, Shah B. Enhancing motor skill learning with transcranial direct current stimulation-a concise review with applications to stroke. Front Psychiatry. 2012; 3: 66 .

44. Nitsche M, Fricke K, Henschke U, Schlitterlau A, Liebetanz D, Lang N, et al. Pharmacological modulation of cortical excitability shifts induced by transcranial direct current stimulation in humans. Adv Physiol Educ. 2003; 553(1): 293-301.

45. Hess G, Aizenman CD, Donoghue JP. Conditions for the induction of long-term potentiation in layer II/ III horizontal connections of the rat motor cortex. J 
Neurophysiol. 1996; 75(5): 1765-78.

46. Lindenberg R, Nachtigall L, Meinzer M, Sieg MM, Flöel A. Differential effects of dual and unihemispheric motor cortex stimulation in older adults. J Neurosci.. 2013; 33(21): 9176-83.

47. Chen T-Y, Hwang I-S, Chang G-C. Effects of transcranial direct current stimulation on balance in healthy adults. Physiotherapy. 2015; 101: e229.
48. Steiner KM, Enders A, Thier W, Batsikadze G, Ludolph N, Ilg W, et al. Cerebellar tDCS does not improve learning in a complex whole body dynamic balance task in young healthy subjects. PloS one. 2016; 11(9): e0163598.

49. Jeffery DT, Norton JA, Roy FD, Gorassini MA. Effects of transcranial direct current stimulation on the excitability of the leg motor cortex. Exp Brain Res. 2007; 182(2): 281-7. 